\title{
IMPACT OF GAP FILLING ON QUALITY OF ROAD NETWORKS
}

\author{
M. Maboudi ${ }^{{ }^{*},}$ J. Amini ${ }^{2}$, M. Gerke ${ }^{1}$ \\ ${ }^{1}$ Institute of Geodesy and Photogrammetry, Technische Universität Braunschweig, Germany- (m.maboudi, m.gerke)@tu-bs.de \\ ${ }^{2}$ School of Surveying and Geospatial Engineering, College of Engineering, University of Tehran, Iran- jamini@ut.ac.ir
}

KEY WORDS: Road network, Gap filling, Topology, Quality assessment, Tensor voting

\begin{abstract}
:
High quality and updated road network maps provide important information for many domains. Many small segments appear on the road surface in VHR images. Most road extraction systems have problem in extraction of these small segments and usually they appear as gaps in the final extracted road networks. However, most approaches skip filling these gaps. This is on account of the fact that usually overall length of the missing parts of the road extraction results is very short relative to the total length of the whole road network. This leads to an indiscernible impact of filling these gaps on geometrical quality criteria. In this paper, using two different VHR satellite datasets and a gap-filling approach which is based on tensor voting, we show that utilizing an effective road gap filling can result in a quite tangible topological improvement in the final road network which is highly demanded in many applications
\end{abstract}

\section{INTRODUCTION}

Due to the importance of updated road network database, automated road extraction is an important study area in remote sensing. High quality and updated road network maps provide important information for many domains, e.g., driving assistance systems, transportation management, smart city planning, and Geographic Information System (GIS). There is a large body of literature about road extraction from very high resolution (VHR) images (Mena, 2003; Quackenbush, et al., 2013; Wang et al., 2016; Maboudi et al., 2017). However, to the best of our knowledge, there are few comprehensive studies focusing on filling gaps of the extracted road network and analyzing its effect on the overall quality of the network.

Many small segments appear on road surface in VHR images which are originated by noise and small objects like road markings, cars, adjacent objects like buildings, trees and their shadows. Most road extraction systems have problem in extraction of these small segments and usually they appear as gaps in automatically extracted road networks. There are some attempts to improve the road extraction systems in order to recover or fill these gaps.

Employing mathematical morphology, perceptual grouping, active contours, and multi-scale processing followed by topology transmission (Zeng et al., 2015) are the most frequent techniques which are used for this reason. However, most road extraction systems skip this step. This is on account of the fact that usually the length of the missing parts i.e., gaps is very short relative to the total length of the network, which leads to an indiscernible impact of filling these gaps on geometrical quality criteria such as completeness, correctness and quality. Nevertheless, even small gaps may have a strong destructive effect on topology of the network (Wiedemann, 2003, Wegner et al., 2015) . Existence of small gaps which their elimination in our experiment shows less than $4 \%$ improvement in geometric quality indices, may lead to lengthy detours and affect the topology of the road network, seriously.

Roads connect different places. This inherent functionality of the roads which is a unique property w.r.t other objects like buildings and vegetation should be considered in road extraction/updating systems. This property is considered as connectedness in (Wegner et al., 2015) and topological quality of the extraction results is investigated, thoroughly.
In (Mosinska et al., 2018) a deep learning approach is presented for detection of curvilinear structures.

The authors add a topological term to the loss of the neural network that accounts for topology of curvilinear structures like roads. Radon transformation is used in (Matkan et al., 2012) in order to detect the gaps in road network which are extracted from LiDAR raster data. Then the detected discontinuities are filled using spline interpolation. The number of gaps within the dataset which are filled is used for evaluation.

Filling gaps of the network of linear objects is also an interesting topic in various disciplines like medical image processing and plant breeding and phenotyping. A tensor voting based approach is introduced in (Risser, et al., 2008) for merging undesirable gaps in 3D images of tubular structures in micro-vascular networks. Number of detected gaps, their length, as well as perceptual visual quality of the network are used to evaluate the efficiency of the proposed approach. Recently, (Chen et al., 2018) proposed a deep neural network to recover gaps from disconnected plants' root segments. The authors utilized a fully supervised encoder-decoder deep convolutional neural network $(\mathrm{CNN})$ in order to recover the missing parts of the network.

Different approaches for road extraction from various types of images are developed and geometrical quality of the results are evaluated. There are some attempts to fill the gaps in the extracted road network. Furthermore, in some research the importance of topological quality of the road network is emphasized. However, the effect of gap filling on the quality of the extracted network is not analyzed yet which is the main focus of this paper.

\section{ROAD EXTRACTION AND GAP FILLING}

The main aim of this paper which is analyzing the effect of gap filling on quality of the extracted network depends neither on a specific road extraction algorithm nor on a gap filling approach. However, in the two following parts of this section, we briefly mention a road extraction approach as well as a gap filling method which are used in our experiment.

\subsection{Road extraction}

In this research we employ an object based road extraction approach which relies on a road model and analyses the image

\footnotetext{
* Corresponding author
} 
objects characteristics (Maboudi et al., 2018). This fuzzy object based road extraction approach exploits an integration of fuzzy reasoning in a bio-inspired swarm intelligence algorithm, namely object based ant colony optimization (ACO), for extraction of road network. First, guided filtering as an edgepreserving smoothing algorithm is utilized to smooth the multispectral VHR images in order to reduce the road surface heterogeneity. Next, the smoothed images are segmented exploiting a bottom up region-merging algorithm (Baatz and Schäpe, 2000) to generate image objects. Then, considering an effective road model, some structural, spectral, and textural road descriptors are calculated and integrated in a fuzzy inference system (FIS) to assign a road membership value to each object. By injecting this information as heuristic information to ACO, it can discriminate the road objects, efficiently. Finally utilizing the tensor voting approach (Kang and Medioni, 2005) most gaps in the extracted road network are filled.

\subsection{Gap filling}

Tensor voting as a non-iterative algorithm is an efficient framework for the robust inference of perceptually salient information from noisy and sparse data (Kang and Medioni, 2005). According to tensor voting (TV) framework and by applying spectrum theorem, any 3D tensor can be expressed as a linear combination of ball, plate and stick tensors as follows:

$$
\begin{gathered}
T=T_{\text {Stick }}+T_{\text {Plate }}+T_{\text {Ball }}= \\
\left(\lambda_{1}-\lambda_{2}\right) e_{1} e_{1}^{T}+\left(\lambda_{2}-\lambda_{3}\right) \sum_{i=1,2} e_{i} e_{i}^{T}+\lambda_{3} \sum_{i=1,2,3} e_{i} e_{i}^{T}
\end{gathered}
$$

Stick tensor of a voter token as the fundamental part of voting propagates its information and communicate with other tokens during a voting process (Maboudi et al., 2016) which is illustrated in Figure 1.

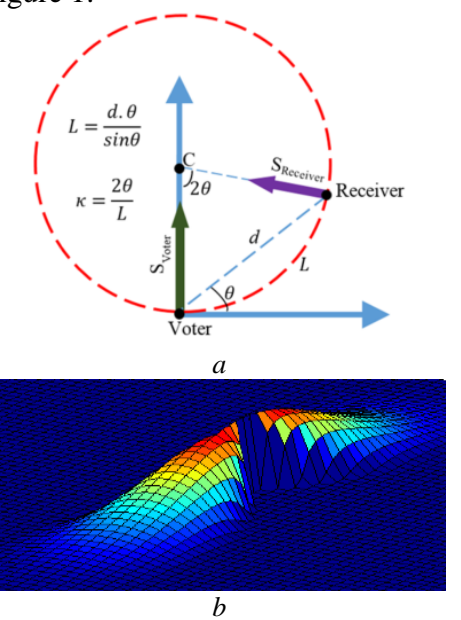

Figure 1. Stick voting. a) Vote casting from "Voter" token to "Receiver" token (Kang and Medioni, 2005) ; b) voting field.

The strength of the received vote at receiver position could be expressed as:

$$
D F(L, k)=-\exp \left(\left(L^{2}+c k^{2}\right) / \sigma^{2}\right)
$$

where $L$ and $k$ are visible in Figure 1a , $\sigma$ is the scale of voting which determines the range within which tokens can influence each other and $c$ is a parameter which is a function of $\sigma$ and controls the relative weight of path length and curvature. The interested reader can find more details of tensor voting in (Maboudi et al., 2016, Kang and Medioni, 2005, Henderson and Linton, 2009).

\subsection{Quality assessment}

\subsubsection{Geometric criteria}

In our experiments in next section, geometric quality of the road network is investigated using the so-called "buffer method" and three very popular metrics, namely geometric completeness, correctness and quality, which are used in many papers are adopted. According to the definition of these metrics (Wiedemann, 2003) for comparing a network with a ground truth (reference network), every portion of one network within a given distance of the other network is considered as matched and then the metrics are defined as follows:

$$
\begin{gathered}
\text { Completeness }=\frac{\text { lenght of matched reference }}{\text { lenght of reference }} \times 100 \\
\text { Completeness }=\frac{\text { lenght of matched extraction }}{\text { lenght of extraction }} \times 100 \\
\text { Quality }=\frac{\text { lenght of matched extraction }}{\text { lenght of ext. +length of unmatched ref. }} \times 100
\end{gathered}
$$

Using the above mentioned criteria the geometric quality of the extracted road network can be evaluated. However, for a thorough evaluation of the quality of a road network, geometric criteria alone are not enough because of their inability to reflect the possible imperfections in network properties (topology) of the results (Wegner et al., 2015, Maboudi et al., 2018).

\subsubsection{Topological criteria}

In order to calculate the topological criteria, firstly, two sets of points are considered in reference network. These include some equidistance points on the reference network and all junctions and endpoints. Union of these two sets which provide the reference points $(\mathrm{R})$ are depicted in Figure 2b. For all pairs in R, which are connected in the reference network, it is checked if their homologous nodes $R^{\prime}$ are connected in the extracted road network. If the connections in $R$ are called $\left(E_{R}\right)$ and the connections in $R$ which have homologous connections in $R^{\prime}$ are called $E_{R, R^{\prime}}$, then the topological completeness is defined as:

$$
\operatorname{Comp}_{\text {Top }}=\frac{n_{E_{R, R^{\prime}}}}{n_{E_{R}}} \times 100
$$

where $n$ indicates the cardinality of each set. The optimum value for the topological completeness is $100 \%$. Nevertheless, possible fragmentation of the extracted network with respect to the reference network decreases this value.

In order to compute the topological correctness, analysis is conducted in the other way around. It means if the union of all junctions, endpoints, and equidistance points in the extracted network is called $D$ and its counterpart in reference is called $D^{\prime}$, the topological correctness is defined as:

$$
\operatorname{Corr}_{T o p}=\frac{n_{E_{D, D^{\prime}}}}{n_{E_{D}}} \times 100
$$

\section{EXPERIMENTAL RESULT}

\subsubsection{First experiment}

The first dataset is a worldview-2 multispectral image from part of San Clemente, California, USA, which was made publicly available by DigitalGlobe. Most of the roads in this part of the city are not straight and have a curved shape which makes it challenging for some road extraction algorithms. Figure $2 \mathrm{a}$ shows the visible bands of Worldview-2 image of study area with overlaid road network extraction (cf. section 2.1) result before gap filling. 


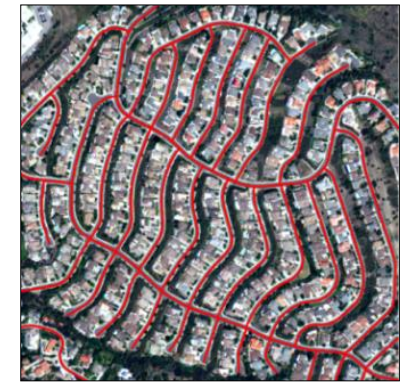

$a$

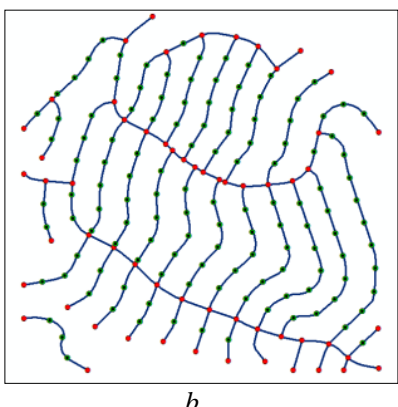

$b$
Figure 2. First study area. a) Road network extraction result overlaid on the RGB bands of the worldview-2 image; b) Selected points for topological evaluation of the extracted road network. Green points are some equidistance points and red points are junctions and endpoints.

The main reason that we used this dataset in our experiment is that different colors of the road surface in most junctions lead to discontinuities in the extracted roads network. This characteristic which is depicted in Figure 3 clearly reveals the difference between geometric quality and topological quality of the extracted road network which is aimed in this paper.

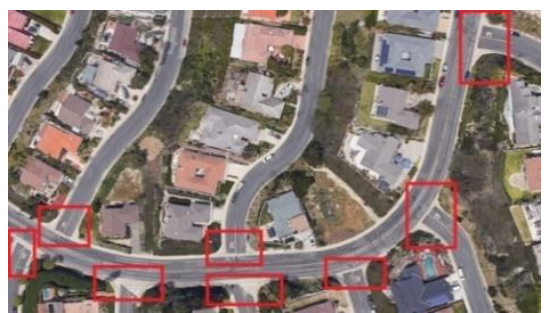

Figure 3. Different colors of road surface in junctions may lead to discontinuities in the extracted roads network.

Employing a road extraction approach (cf. Section2.1) the road network of the study area is extracted and different geometric and topological criteria (cf. section 2.3) are listed in Table 1. Same criteria are reported after filling the gaps of the network.

\begin{tabular}{|l|l|c|c|}
\hline \multicolumn{2}{|c|}{ Criteria (\%) } & Road Extraction & After Gap Filling \\
\hline \multirow{3}{*}{ Geometric } & Completeness & 91 & 92.7 \\
\cline { 2 - 4 } & Correctness & 93 & 93.1 \\
\cline { 2 - 4 } & Quality & 85 & 87 \\
\hline \multirow{2}{*}{ Topological } & Completeness & 43 & 89 \\
\cline { 2 - 4 } & Correctness & 99 & 99 \\
\hline
\end{tabular}

Table 1. Evaluation of extracted network in the first experiment

Despite the ability of tensor voting in filling the gaps of the extracted network, it can easily be understood from Table 1 that geometric improvement of the results after gap filling is not considerable. This is on account of the fact that the length of the missing parts is very small relative to the total length of the network. However, comparing the topological criteria reveals that exploiting a successful gap filling approach improved the topological quality of the network, significantly.

As stated before (cf. Figure 3), different colors of the road surface in most junctions caused numerous small but topologically important gaps in the network which lead to a slump in topological completeness of the network. Consequently, employing gap filling to amend these gaps caused a great improvement of the results, which are recognized by topological completeness criterion.

\subsubsection{Second Experiment}

For this experiment we used three VHR image patches. Each patch covers an area of $1000 \times 1000 \mathrm{~m}$. Spatial resolution of the multi-spectral bands is $4 \mathrm{~m}$ and a panchromatic band with $1 \mathrm{~m}$ resolution is also delivered. Road extraction results as well as the ground truth data which is used in our experiment for quality assessment are shown in Figure 4.
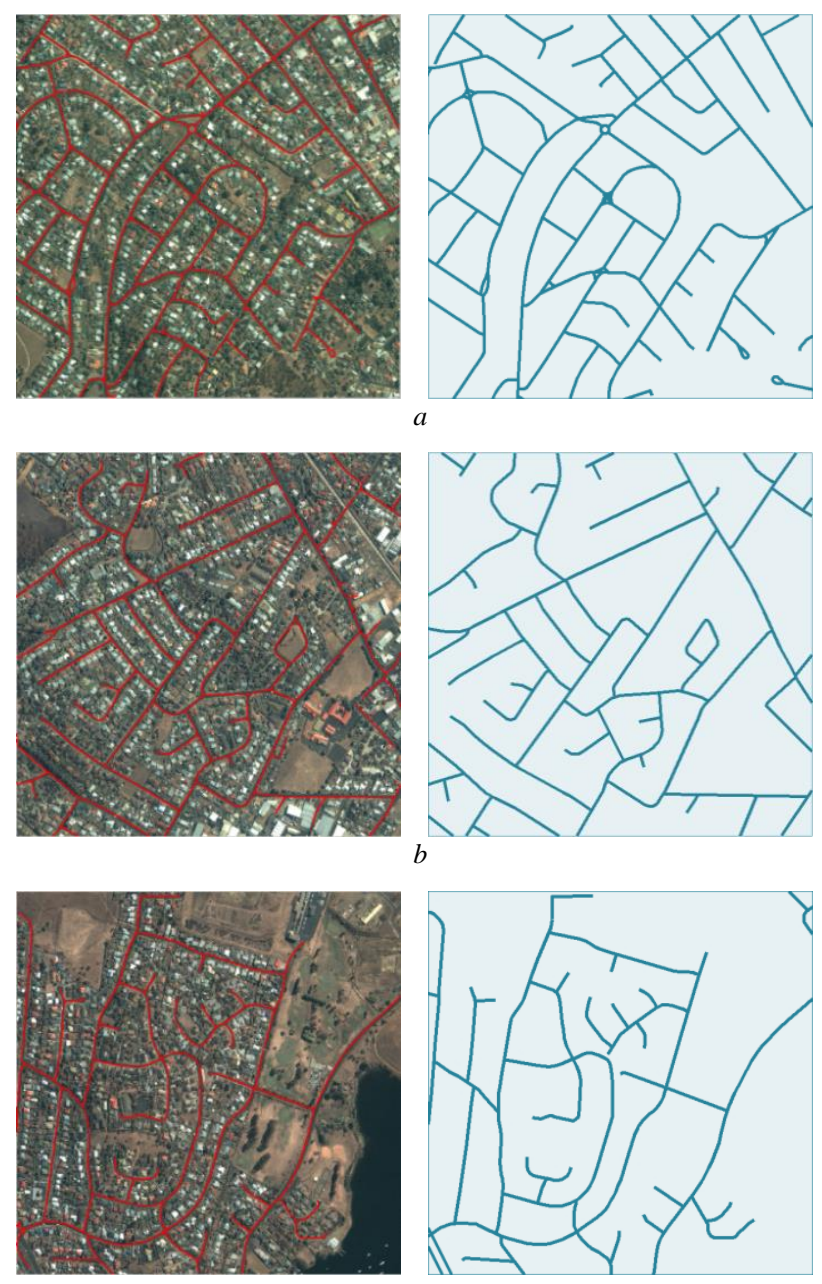

Figure 4. Second dataset. Road extraction results are depicted in left column and right column images illustrate the ground truth.

By visual inspection of the results in left column of Figure 4 and also by computing the initial geometric performance criteria which are listed in Table 2, geometric usefulness of the extracted road network could be verified. However, existence of gaps is inevitable. Some small gaps in the extracted road network are illustrated in Figure 5. Existence of these gaps affects the overall topological quality of the extracted road network.
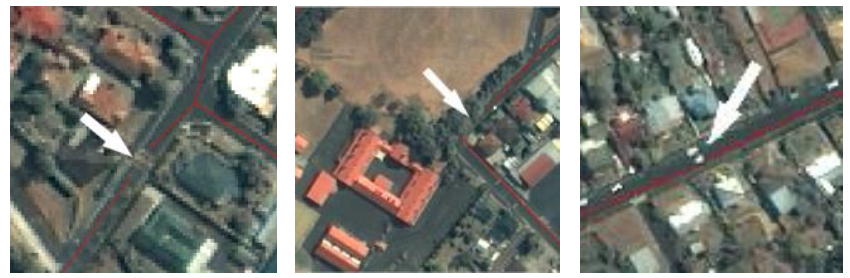

Figure 5. Some small gaps in the extracted road network.

We utilized the same approach that we used in first experiment to extract the roads and fill their gaps. Table 2 shows the evaluation results before and after road gap filling. 


\begin{tabular}{|c|c|c|c|c|c|c|c|}
\hline \multirow{2}{*}{} & \multicolumn{2}{|c|}{ Figure 4a } & \multicolumn{2}{c|}{ Figure 4b } & \multicolumn{2}{c|}{ Figure 4c } \\
\cline { 3 - 8 } & $\begin{array}{c}\text { Initial } \\
\text { Result }\end{array}$ & $\begin{array}{c}\text { After } \\
\text { Gap } \\
\text { Filling }\end{array}$ & $\begin{array}{c}\text { Initial } \\
\text { Result }\end{array}$ & $\begin{array}{c}\text { After } \\
\text { Gap } \\
\text { Filling }\end{array}$ & $\begin{array}{l}\text { Initial } \\
\text { Result }\end{array}$ & $\begin{array}{c}\text { After } \\
\text { Gap } \\
\text { Filling }\end{array}$ \\
\hline \multirow{3}{*}{ Geometric (\%) } & Fompleteness & 89 & 90 & 86 & 88 & 90 & 90 \\
\cline { 2 - 8 } & Correctness & 93 & 93 & 95 & 96 & 91 & 91 \\
\cline { 2 - 8 } & Quality & 84 & 84 & 82 & 85 & 82 & 82 \\
\hline \multirow{2}{*}{ Topological } & Completeness & 89 & 99 & 86 & 98 & 84 & 96 \\
\cline { 2 - 8 } & Correctness & 91 & 93 & 96 & 97 & 98 & 98 \\
\hline
\end{tabular}

Table 2. Evaluation of extracted network in the second experiment

As it can be seen from Table 2, the most sensible improvement by road gap filling is related to topological completeness. It is in line with our expectation. Because, the length of the missing parts is usually very short w.r.t the total length of the network. Furthermore, because of the nature of the gaps, they are related to false negative parts of the results. Hence the geometric correctness may be affected by network gaps, rarely and slightly. Furthermore, based on the definition of the topological completeness, missing parts of the network may decrease the topological completeness, severely. The extreme cases could be the gaps in those parts of the network which separate one or some parts of the network from the others, completely.

\section{CONCLUSION}

Experimental results on our datasets show the improvement of at most $4 \%$ in geometrical quality of the extracted road network. This improvement, especially when the computational load of the tensor voting algorithm is considered into account, may not be convincing for embedding gap filling step to road extraction systems. However, analyzing the topological quality of the extracted road networks shows that existence of gaps in a road network, even with a high geometric quality, may lead to a low level of topological quality. Hence, a tensor voting based road gap filling is utilized as a remedy for this problem and its topological effectiveness is measured. The achieved results indicate that the topological quality of the results is improved significantly.

The most important take home message of this paper is that exploiting a road gap filling approach in a road extraction system may deliver a non-significant geometrical benefit. Nonetheless, utilizing an effective road gap filling can result in a quite tangible topological improvement. Moreover, the presented strategy can be used in assessment of road gap filling approaches which is highly demanded. The authors also urge that because of the inherent network structure of the road network which is different from other man-made objects like buildings, topology of the network should be addressed in the evaluation of road extraction methods. Our analysis can be adopted for other linear structures like rivers and similar objects in medical images.

Image inpainting seems to be a very promising approach for filling the gaps in network structures like roads. Moreover, topology transfer in multi-scale VHR image processing could be considered as a topic for future research.

\section{ACKNOWLEDGMENTS}

The authors would like to thank ISPRS, Space Imaging LLC, and DigitalGlobe for providing the satellite images.

\section{REFERENCES}

Baatz, M. and Schäpe, A. (2000) 'Multiresolution Segmentation: an optimization approach for high quality multiscale image segmentation', in Angewandte Geographische Informations- Verarbeitung XII. Salzburg, Austria, pp. 12-23.
Chen H., Valerio Giuffrida M., Doerner P., and Tsaftaris S. A. (2018) 'Root Gap Correction with a Deep Inpainting Model', in Computer vision problems in plant phenotyping, Newcastle,UK.

Henderson, T. C. and Linton, T. (2009) 'Raster map image analysis', in Proceedings of the International Conference on Document Analysis and Recognition, ICDAR, pp. 376-380.

Kang, S. B. and Medioni, G. (2005) Emerging topics in computer vision. Prentice Hall.

Maboudi M., Amini J., Hahn M., and Saati M. (2016) 'Road Network Extraction from VHR Satellite Images Using Context Aware Object Feature Integration and Tensor Voting', Remote Sensing. 8(8), p. 637.

Maboudi M., Amini J., Hahn M., and Saati M. (2017) 'Objectbased road extraction from satellite images using ant colony optimization', International Journal of Remote Sensing, 38(1), pp. 179-198.

Maboudi M., Amini J., Malihi S, and Hahn M. (2018) 'Integrating fuzzy object based image analysis and ant colony optimization for road extraction from remotely sensed images', ISPRS J. of Photog. and Remote Sensing, 138, pp. 151-163.

Matkan A. , Hajeb M., Eslami M., Pourali H., and Sadeghian S. (2012) 'Gap Filling in Road Extraction Using Radon Transformation', in ISPRS Annals of Photogrammetry, Remote Sensing and Spatial Information Sciences, pp. 47-52

Mena, J. B. (2003) 'State of the art on automatic road extraction for GIS update: a novel classification', Pattern Recognition Letters, 24(16), pp. 3037-3058.

Mosinska, A. Márquez-Neila P., Kozi'nski M. K., and Fua P. (2018) 'Beyond the Pixel-Wise Loss for Topology-Aware Delineation', in CVPR. Salt Lake City, US, pp. 3136-3145.

Quackenbush, L. J., Im, J. and Zuo, Y. (2013) 'Road Extraction: A Review of LiDAR-Focused Studies', in Remote Sensing of Natural Resources. CRC Press, pp. 155-170.

Risser, L., Plouraboue, F. and Descombes, X. (2008) 'Gap Filling of 3-D Microvascular Networks by Tensor Voting', IEEE Transactions on Medical Imaging, 27(5), pp. 674-687.

Wang W. , Yang N., Zhang Y., Wang F., Cao T., and Eklund P., (2016) 'A review of road extraction from remote sensing images', J. of Traffic and Transp. Eng., 3(3), pp. 271-282.

Wegner, J. D., Montoya-Zegarra, J. A. and Schindler, K. (2015) 'Road networks as collections of minimum cost paths', ISPRS Journal of Photog. and Remote Sensing.108, pp. 128-137.

Wiedemann, C. (2003) 'External Evaluation of Road Networks', in ISPRS Archives. Munich, Germany, pp. 93-98.

Zeng, C., Bird S., Luce J., Wang J. (2015) 'A Natural-RuleBased-Connection (NRBC) Method for River Network Extraction from High-Resolution Imagery', Remote Sensing, 7(10), pp. 14055-14078 